\title{
Measurement of Carbon Dioxide Column via Space Borne Laser Absorption
}

\author{
William S. Heaps \\ NASA Goddard Space Flight Center \\ Greenbelt, Md 20771 \\ 301-286-5106 \\ William.S.Heaps@nasa.gov
}

In order to better understand the budget of carbon dioxide in the Earth's atmosphere it is necessary to develop a global high precision understanding of the carbon dioxide column. In order to uncover the "missing sink" that is responsible for the large discrepancies in the budget as we presently understand it calculation has indicated that measurement accuracy on the order of $1 \mathrm{ppm}$ is necessary. Because typical column average $\mathrm{CO}_{2}$ has now reached $380 \mathrm{ppm}$ this represents a precision on the order of $.25 \%$ for these column measurements. No species has ever been measured from space at such a precision. In recognition of the importance of understanding the $\mathrm{CO}_{2}$ budget in order to evaluate its impact on global warming the National Research Council in its decadal survey report to NASA recommended planning for a laser based total $\mathrm{CO}_{2}$ mapping mission in the near future.

The extreme measurement accuracy requirements on this mission places very strong requirements on the laser system used for the measurement. This work presents an analysis of the characteristics necessary in a laser system used to make this measurement. Consideration is given to the temperature dependence, pressure broadening, and pressure shift of the $\mathrm{CO}_{2}$ lines themselves and how these impact the laser system characteristics

Several systems for meeting these requirements that are under investigation at various institutions in the US as well as Europe will be discussed.

Keywords: Greenhouse, Carbon dioxide, Laser, LIDAR,

\section{INTRODUCTION}

$\mathrm{CO}_{2}$ is the most prevalent known anthropogenic greenhouse gas. Its concentration has increased by more than $95 \mathrm{ppm}$ in the last 150 years ${ }^{1}$. The majority of $\mathrm{CO}_{2}$ variability occurs in the lower atmosphere $(\sim 1000$ to 800 mbar). The natural geographic distribution and temporal variability of $\mathrm{CO}_{2}$ sources and sinks however are still not well understood ${ }^{2,3}$ (Figure 1). Satellite instruments show promise for identifying sources and sinks globally, but typically measure the total atmospheric column. Since sources and sinks at the surface represent a small perturbation to the total column, a precision of better than $1 \%$ is required. To quantify the carbon cycle dynamics, to help predict climate change and to meet the stringent performance requirements new monitoring instruments are needed. 


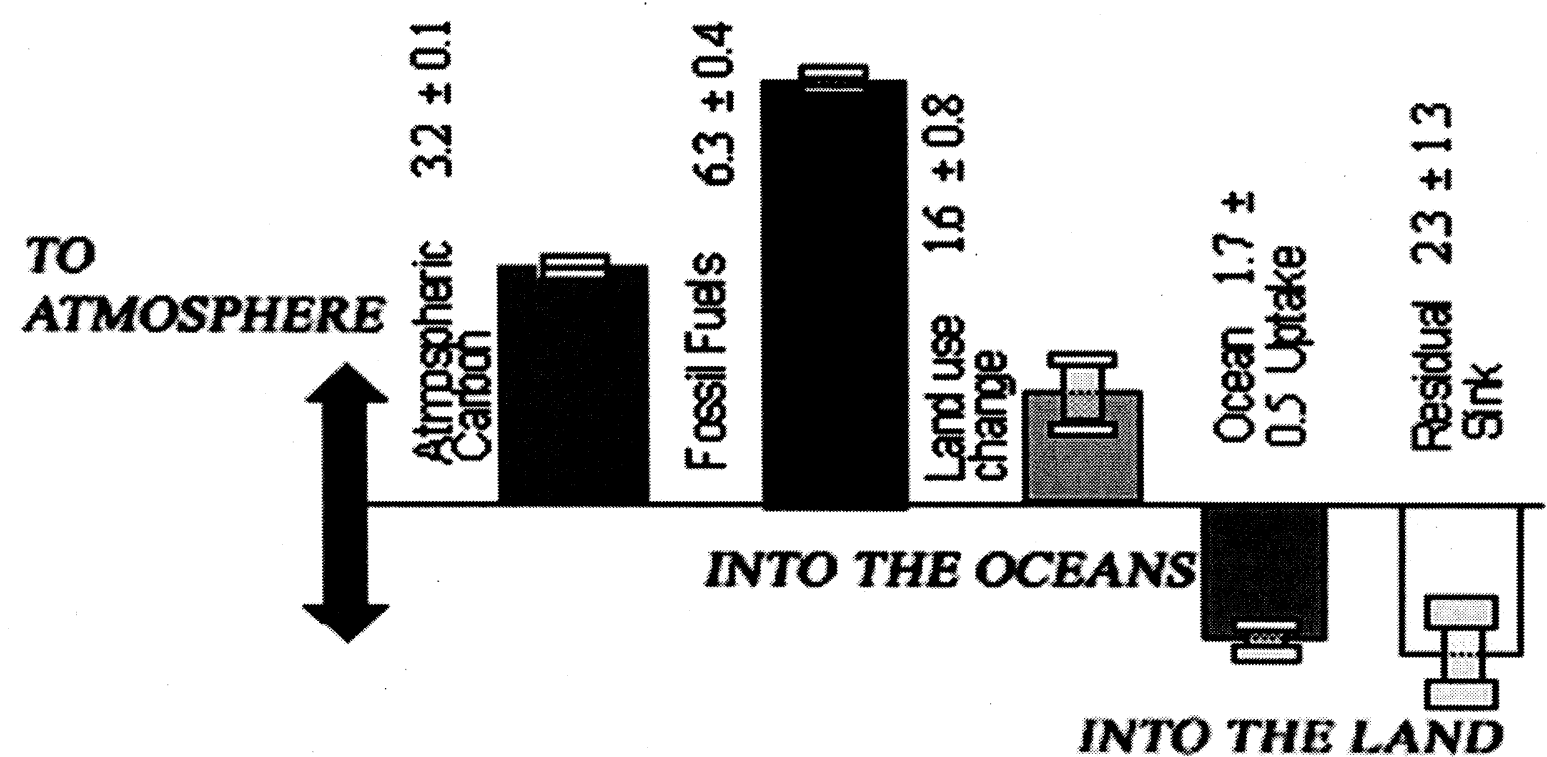

Figure 1. Shown are $\mathrm{CO}_{2}$ sources and sinks in Petagrams $\left(10^{15}\right.$ grams) carbon/year plus atmospheric accumulation. Uncertainties are highest for land use change, oceanic uptake and the residual (missing) sink. The numbers in the figure are from table 3.3 in IPCC 2001 report. $^{4}$

Solutions to the questions and uncertainties regarding $\mathrm{CO}_{2}$ sources and sinks are generally thought to be data limited. The observational foundation of our knowledge of atmospheric carbon processes comes from ground network and satellite measurements which includes the long term, in situ $\mathrm{CO}_{2}$ measurement program led by the NOAA.. The in situ measurements are very precise (uncertainties on the order of $0.1 \mathrm{ppm}$ ) and accurate, but are necessarily limited in time and space and do not measure the whole atmospheric column. Space-based $\mathrm{CO}_{2}$ column measurements with $1 \mathrm{ppm}$ precision were predicted to reduce inferred $\mathrm{CO} 2$ flux uncertainties of annual mean fluxes from greater than $1.2 \mathrm{GtC}$ region-1 year-1 to less than $0.5 \mathrm{GtC}$ region-1 year-1 when averaged over the annual cycle. ${ }^{1}$

Measurement of the total column of $\mathrm{CO}_{2}$ from space at the requisite precision is a formidible task. No satellite measurement of a column has ever approached this level of precision. Nevertheless a number of methods to make this measurement have been proposed over the last few years including passive measurements such as the Orbiting Carbon Observatory (OCO) instrument that NASA proposes to fly next year and GOSAT under development by JAXA as well as a number of active (laser based) instruments.

The most difficult aspect of meeting the precision requirements for the passive sensors is determining the path length through the atmosphere in the presence of clouds and scattering aerosols. A path length error on the order of 20 meters is sufficient to produce the $1 \mathrm{ppm}$ error in the column average which is the goal of these systems. The active systems are able to make a better determination of the path length because the source of the light used for the measurement is under control. These systems face other difficulties as well predominantly because they measure at only a single frequency. This means the weighted value of the $\mathrm{CO}_{2}$ cross section at this frequency must be known with great precision as it changes with temperature and pressure along the path. Also the laser must be "locked" to this frequency so that drifts in laser output do not ruin the measurement. This work will address some of these considerations for the laser based instrument. 


\section{SPECTROSCOPY}

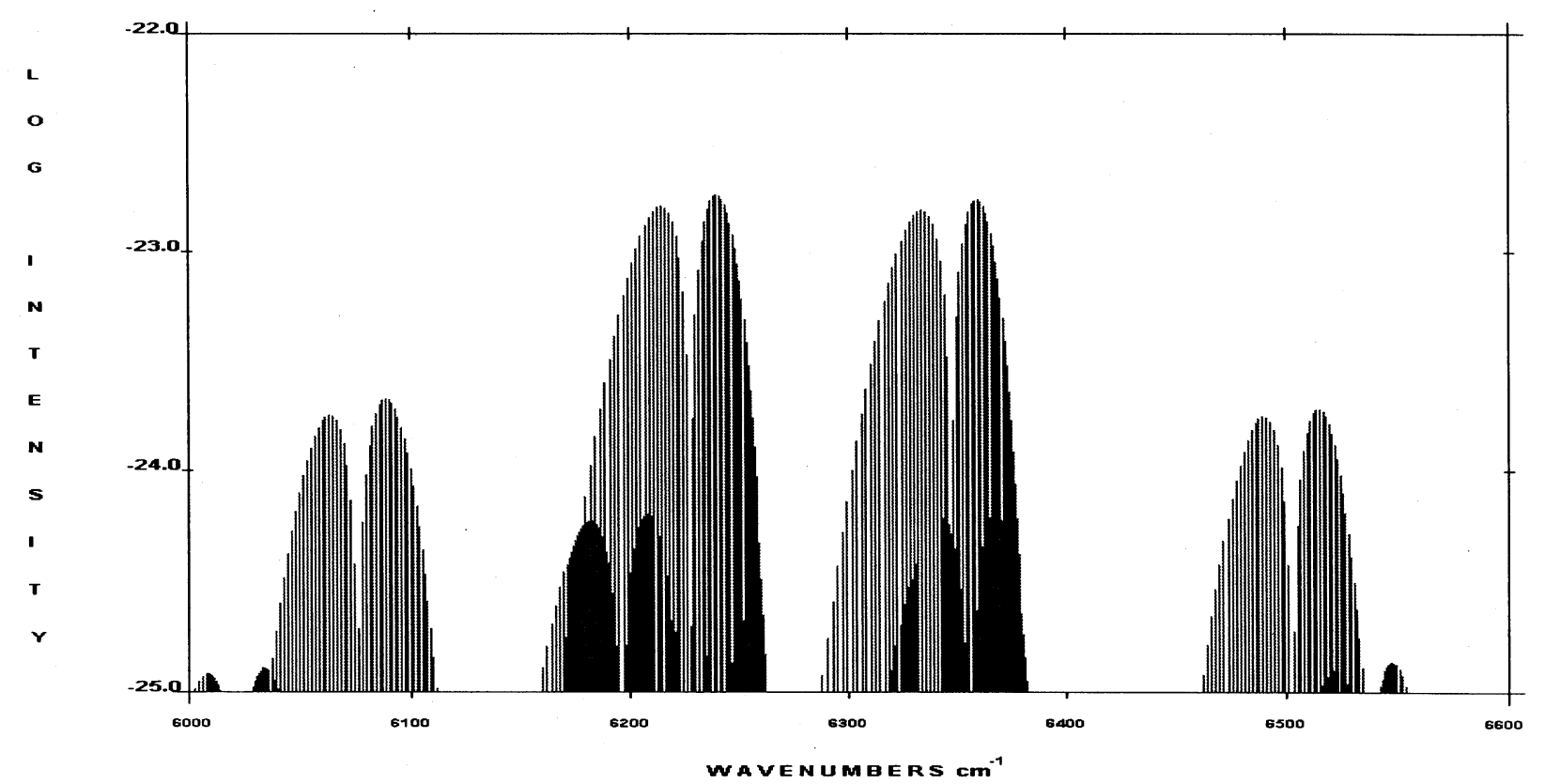

Figure 2. Shown are the linestrengths for the absorption features near 1.6 micron that have been suggested for column measurement from space.

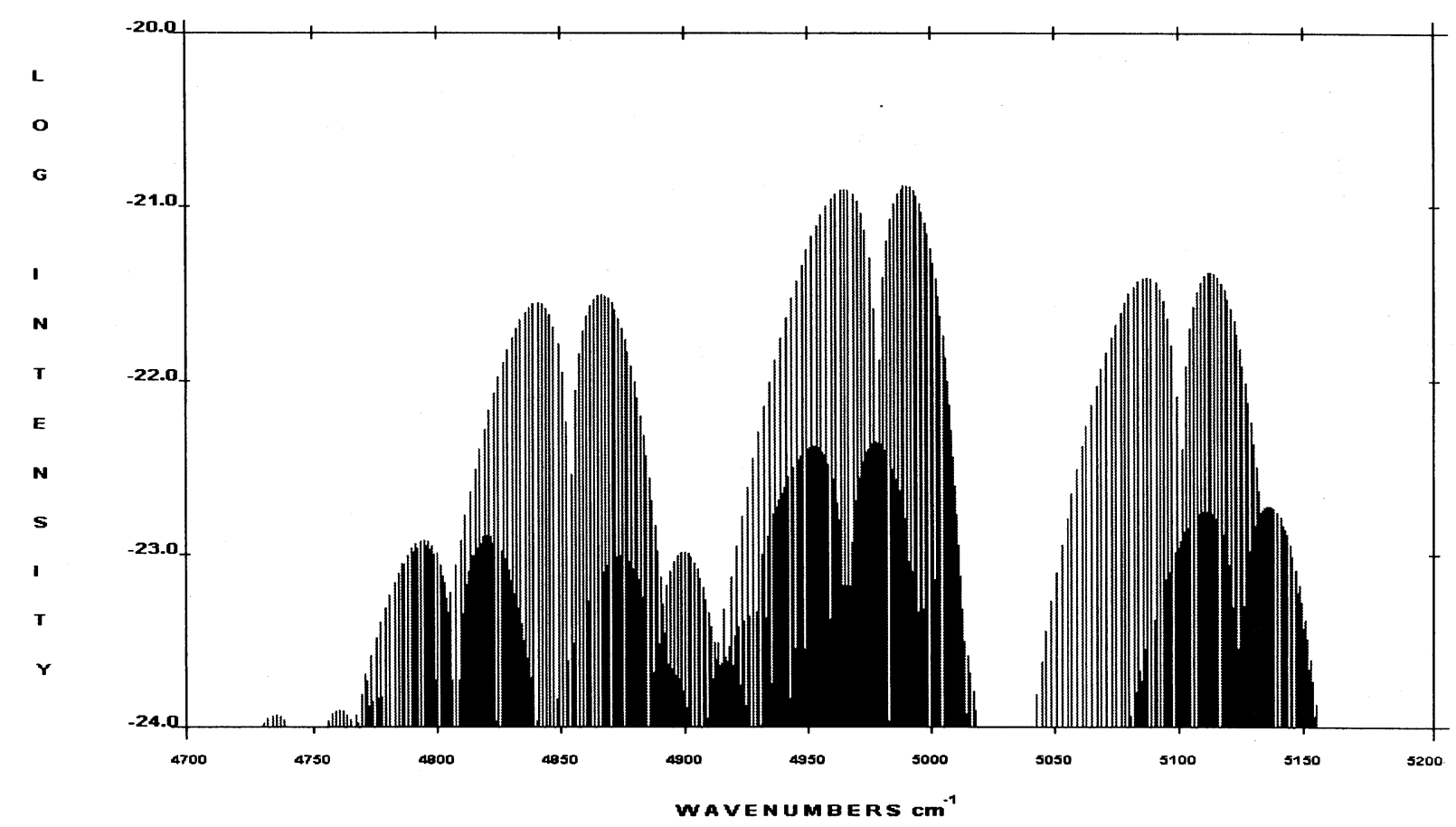

Figure 3. Shown are the line strengths for the absoprtion features near 2.0 micron that have been suggested for column measurement from space. 
Figures 2 and 3 taken from the HITRAN data base show the line strengths of the carbon dioxide absorption features near 1.6 and 2.0 microns that have been proposed for use by space borne laser systems to measure the $\mathrm{CO}_{2}$ column. ${ }^{5}$ The 2.0 micron bands are substantially stronger and indeed the strongest of these lines will absorb almost $90 \%$ of the incident light from a space borne laser during the roundtrip passage through the atmosphere.

The line shapes are rather complex. At the top of the atmosphere the width is dominated by the Doppler effect. Proceeding lower into the atmosphere collisional (pressure) broadening begins to manifest itself to a greater and greater extent. At the same time the actual line strengths are changing with the temperature of the atmosphere and finally a shift in the center frequency also occurs as the result of collisions. ${ }^{6}$

The typical laser line width is substantially narrower than the pressure broadened width of these absorption features. This is to say the laser can easily sample only some portion of the overall absorption line. This can be an asset depending upon one's objective. If for example the problem is to discover regions with anomalous behavior in terms of $\mathrm{CO}_{2}$ production or loss at the surface then one can target surface $\mathrm{CO}_{2}$ by observing changes in the pressure broadened wings of a $\mathrm{CO}_{2}$ line. Since there is only a minimal contribution to the absorption in the wing from the upper atmospheric $\mathrm{CO}_{2}$ the effect of a surface source or sink will be a larger perturbation on the overall column absorption at these wavelengths. This reduces the requirement for $1 \mathrm{ppm}$ precision in order to locate the source or sink. If on the other hand the objective is to quantitatively determine the size of a perturbation on the overall column then this trick will not work as well. This is because the fraction of the total column that is being observed in the wings is difficult to know from a single measurement where the strength of the absorption depends so strongly on the pressure as well as the number density of the carbon dioxide. Figure 4 shows the effect of pressure broadening on a typical $\mathrm{CO}_{2}$ line in the 1.6 micron region.

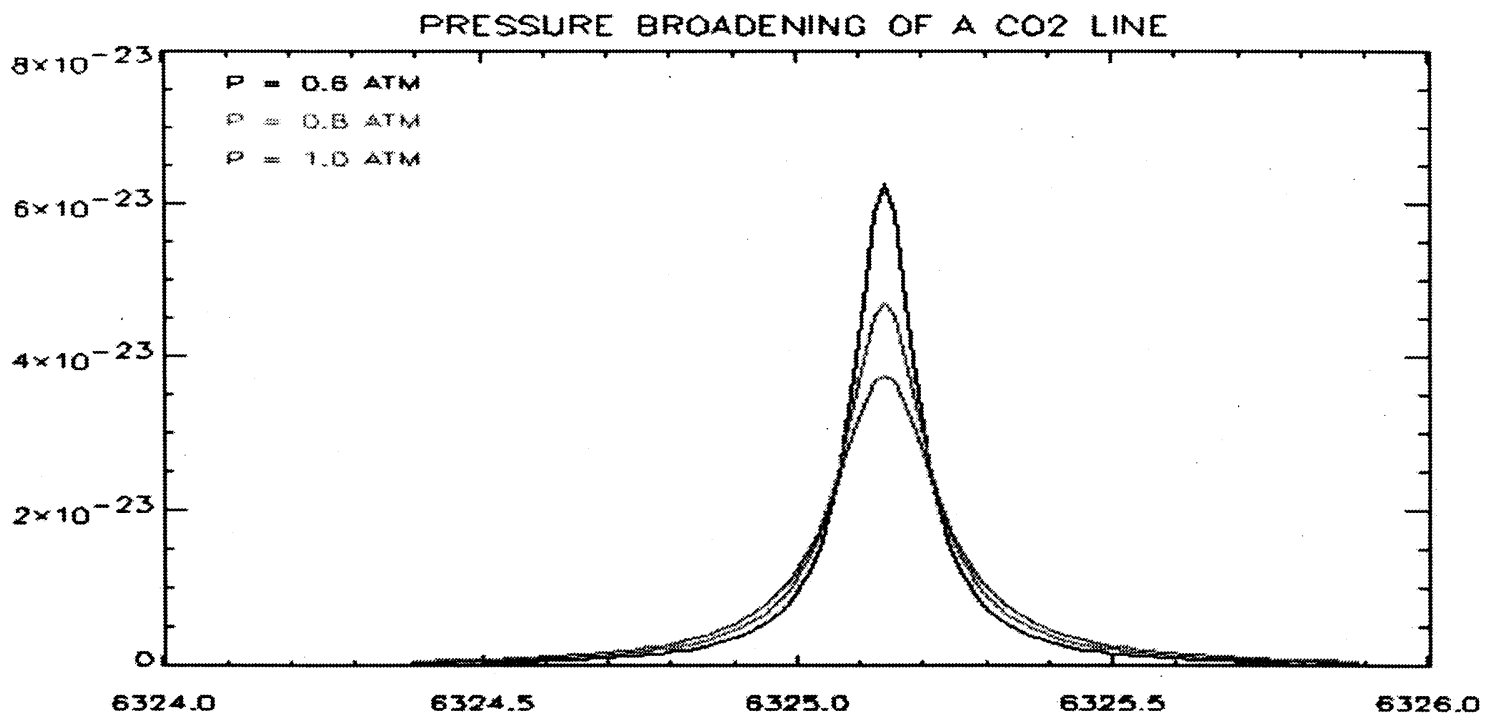

Figure 4. This illustrates the extremely powerful effect of collisional broadening on a typical $\mathrm{CO}_{2}$ line near 1.57 microns.

Temperature also manifests itself on the strength of absorption features through its effect on the population density of the lower level state for one of these absorptions. Lines originating from states arising from the lowest energy levels get weaker with increasing temperature and transitions arising from higher lying levels get stronger. In any manifold of absorptions there are some lines that are affected the least and these are 
probably the best choice for use by a laser system to measure total column. Figure 5 illustrates the effects of temperature on a pair of the less sensitive lines arising from the same lower state energy level. These lines would be good choices for a laser based system.

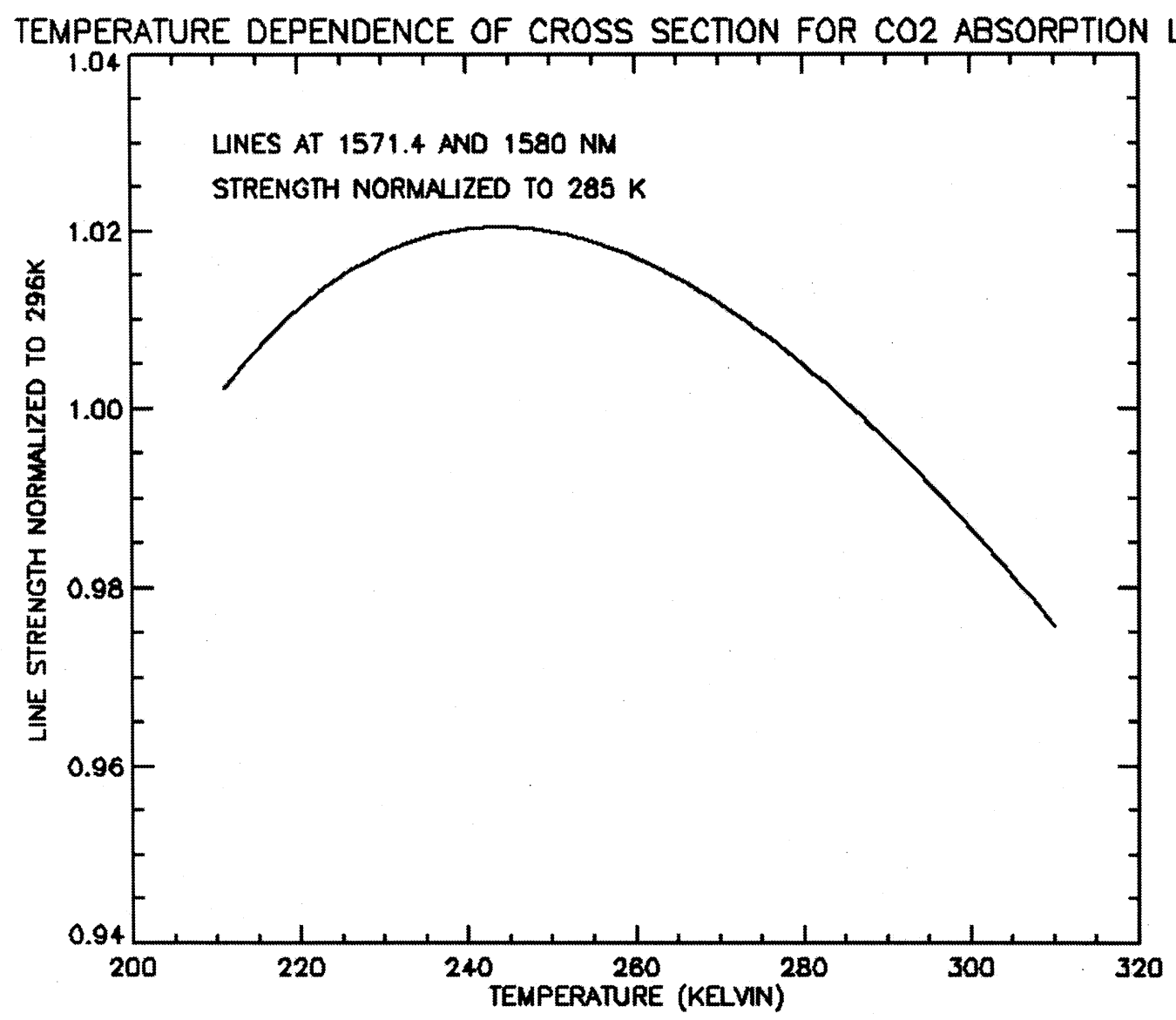

Figure 5. This illustrates the dependence of the line strength of a $\mathrm{CO}_{2}$ absorption line on temperature for a relatively insensitive line. Changes as small as 2 degrees can cause errors in the column measurement as big as $1 \mathrm{ppm}$.

However, even this relatively well behaved line can introduce errors in the column as large as 1ppm for a 2 degree $\mathrm{K}$ change in temperature. Some of the satellite borne instruments that remotely determine temperature from space have temperature errors of this order. ${ }^{7}$ This means that using a single absorption line and relying on meteorological measurements or models to provide the temperature correction may not suffice for the $\mathrm{CO}_{2}$ column measurement. Multiple frequencies are necessary to correct for the effects of variable pressure and temperature on the absorption line strengths and shapes.

A final problem introduced by the spectroscopy of the region is the presence of interference from other atmospheric species. Water vapor is the most significant of these because water vapor concentration is quite variable and water vapor abundance can reach numbers as high as $10,000 \mathrm{ppm}$. Figure 6 shows the spectrum of water vapor for the region from 1.5 to 2.1 microns. Clearly the 1.6 micron region $\left(6100 \mathrm{~cm}^{-1}\right)$ is most favored although the 2.0 micron region $\left(5000 \mathrm{~cm}^{-1}\right)$ also represents a region of weaker water vapor absorption. 


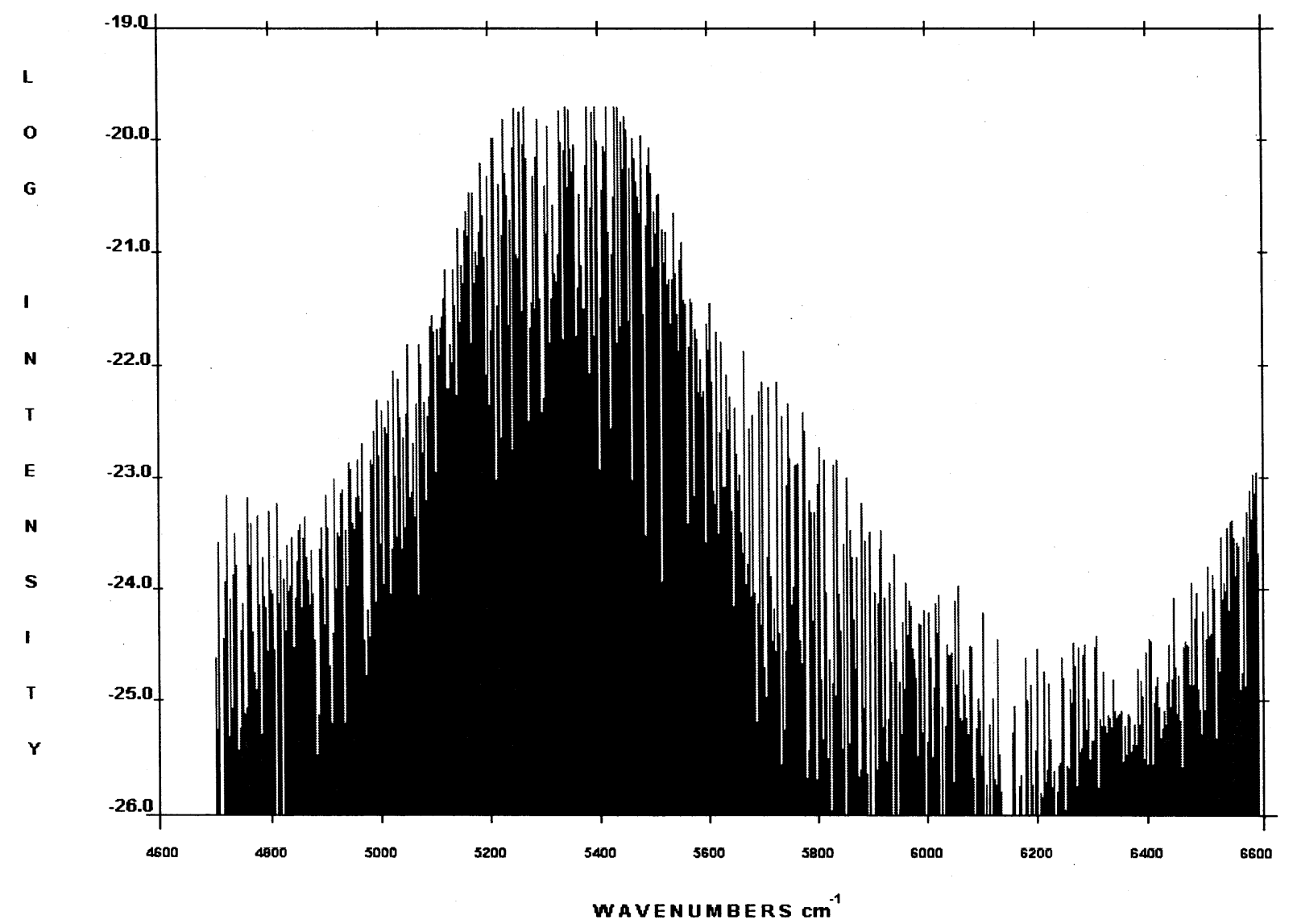

Figure 6. This shows the line strengths of water vapor absorption features in the 1.5-2.1 micron region. Although water vapor absorption features near 1.6 and 2.0 are weaker than $\mathrm{CO} 2$ features water may interfere because it is as much as 50 times more abundant.

In the 1.61 micron region the least temperature sensitive lines are at 6210 and $6242 \mathrm{~cm}^{-1}$. These each have line strengths on the order of $1.5 \mathrm{e}-23$. The closest water vapor lines are at 6209.35 and $6241.69 \mathrm{~cm}^{-1}$ and are respectively 83 and 60 times weaker than the $\mathrm{CO}_{2}$ lines. The water vapor lines are also down by about a factor of 50 from their peak strength in the wings near the center of the $\mathrm{CO}_{2}$ lines. On the other hand the water vapor could be as much as 50 times more abundant than $\mathrm{CO}_{2}$ and if they contribute an absorption as much as one part in 400 then it produces an unacceptable error in the $\mathrm{CO}_{2}$ column. The case for the 1.57 micron region is similar and the 2.0 micron cases appear to be worse. The upshot is that additional care must be exercised in the selection of lines used to measure the $\mathrm{CO}_{2}$ to make sure that water vapor does not introduce an unacceptable error.

Methane is the only other species that has any potential to cause an interference. Methane has some significant lines near 1.6 micron but they should not represent a problem as long as one is careful to stay away from them.

\section{LASER SYSTEMS AND LASER SOUNDERS}

As discussed above the principal wavelengths needed for measuring column $\mathrm{CO}_{2}$ lie in the 1.6 and 2.0 micron regions. A number of approaches have been suggested to generate these wavelengths. For 2.0 microns the 
Ho:Tm laser has been under development for a decade or more. NASA Langley has developed conductively cooled Ho:Tm lasers with output energies in the 1 Joule range. They have also engaged in a considerable amount of testing on the lifetime and survivability of $792 \mathrm{~nm}$ diodes used to pump the Ho:Tm system.

Because of its proximity to 1.55 microns which has been developed extensively for fiber telecommunications a number of groups have proposed that 1.57 and 1.61 microns for $\mathrm{CO}_{2}$ measurement could be generated employing technologies from the telcom industry. Most recently this consists of fiber lasers employing erbium doped glass which are available commercially with high average power and very high standards of manufacturing. It is believed that fiber lasers have been used in space for communication purposes. The problems of fiber lasers and fiber amplifiers for spectroscopic applications is the stimulated Brillouin scattering effect which degrades the line width of the output laser line as output energy is raised. Efforts are under way at a number of laboratories to defeat or reduce the effect of this process.

The workhorse for space borne lasers has been the diode pumped solid state Nd:YAG laser. Lasers of this type were used to map Mars on the MOLA instrument. They are enroute to Mercury for another mapping mission and two instruments are on orbit at present ICESAT and CALIPSO. The output wavelength of Nd:YAG lasers in near 1.06 microns but devices called Optical Parametric Oscillators (OPO) and Optical Parametric Amplifiers (OPA) have been demonstrated to efficiently convert 1.06 micron light to 1.57 micron light for $\mathrm{CO}_{2}$ measurement.

Of course it is also necessary to control the output frequency of the laser light if one is to measure a chemical species using spectroscopic methods. There are a variety of technologies for producing narrow frequency output lasers and "locking" the frequency to a spectroscopic reference. (See for example Eloranta and Razenkov. ${ }^{8}$ ) None of these technologies has been implemented in space to date but at least the methodologies have been demonstrated.

Recently more detailed proposals for space borne $\mathrm{CO}_{2}$ sounders have been put forward by several groups. ${ }^{9-11}$ A proposal from NASA Langley, ITT, and the University of New Hampshire suggests using modulation spectroscopy with a continuous fiber laser to measure $\mathrm{CO}_{2}$ column. The modulation technique provides a way to eliminate interferences among the various lasers operating at different frequencies. On the other hand because there is no laser pulse with this approach timing cannot be used to deduce the path length. One additional pulsed laser will be necessary with this system to identify the presence of cloud or aerosol layers that reflect light back into the instrument before it has reached the ground as well as changes in path caused by terrain.

A group at NASA Goddard has proposed using a set of 1.6 micron fiber laser operating in a pulsed mode to measure the $\mathrm{CO}_{2}$ column. The plan is to transmit each frequency in turn rapidly in order to recover the effects of pressure and temperature on line strength. The disadvantage of this approach is that the satellite moves during the time period that the laser frequencies are cycled so the target reflectivity could change during this period. If the reflectivity changes by more than one part in four hundred the requisite precision is lost.

Finally a French group has proposed a conventional DIAL system operating in the 2 micron region. This system will face the same challenges as the other two.

As a final observation it must be noted that changes in the atmospheric pressure due to weather systems can also cause changes in the $\mathrm{CO}_{2}$ column larger than $1 \mathrm{ppm}$. In order to correct for these effects it has been proposed that simultaneous measurements of oxygen be made as well to permit normalization of the column. Laser systems measuring oxygen will be subject to all the temperature and pressure effects that apply to carbon dioxide. 


\section{CONCLUSIONS}

Improved measurements of carbon dioxide on a global scale continue to be a high priority for increasing our understanding of the process of greenhouse gas induced global change. Laser based instrumentation operating from space appears to be an option for addressing this problem. The advantage of lasers are that they can determine the optical path length for the measurement process very precisely eliminating a serious source of error that may affect passive systems. Lasers can also operate without the need for sunlight and so can make measurements of the full diurnal cycle of $\mathrm{CO}_{2}$ around the whole earth.

The disadvantages of lasers are that they only measure at one distinct and very specific frequency. In order to understand the effects of pressure induced shifts and pressure induced broadening it is probably necessary to measure at three different locations on a carbon dioxide absorption line. In order to understand the effects of temperature on the absorption features it is probably necessary to measure the properties of at least 2 distinct absorption features. To do this means that 6 independent laser sources locked precisely to 6 different frequencies must be flown and must continue to operate simultaneously. In order to measure oxygen at the same time with the same level of precision another 6 lasers are probably required.

NASA has never flown a narrow band frequency locked laser in space. Nor has it flown fiber lasers, Ho:Tm lasers, OPO's or OPA's. NASA has never operated a system in space with 6 or 12 lasers all firing quasi simultaneously and synchronously. Perhaps the need to understand in detail the progress of global warming will provide sufficient impetus to undertake a task of such daunting proportion.

\section{REFERENCES}

1. U.S. Global Carbon Cycle Science Program 2007, Highlights of Recent Research and Plans for FY 2007, Chapter 5 pp.114.

2 Crutzen, P.J., "Geology of mankind," Nature, 415, 23, 2002.

3 Sarmiento J L, Gruber N Sinks for Anthropogenic Carbon, Physics Today, 30-36, 2002.

4 O'Brien D. M., Rayner P. J., "Global observations of the carbon budget 2. CO2 column from differential absorption of reflected sunlight in the $1.61 \mathrm{~mm}$ band of CO2,", Journal of Geophysical Research, 107, 4354, 2002.

5. L. S. Rothman, et al. "The HITRAN Molecular Spectroscopic Database and HAWKS (HITRAN Atmospheric Workstation): 1996 edition," J. Quant. Spectros. Radiat. Transfer 60, 665-710 (1998).

6. Sakaizawa et al., "The pressure shift of carbon dioxide for the on-line wavelength of $1.6 \mathrm{pm} \mathrm{CO}_{2}$ DIAL," presented at 23rd International Laser Radar Conference, Nara, Japan, 24 July, 2006.

7. D. Sun and R. T. Pinker, "Implementation of GOES-based land surface temperature diurnal cycle to AVHRR,"International Journal of Remote Sensing, Vol. 26, No. 18, 20 September 2005, 3975-3984.

8. E. W. Eloranta and I. A. Razenkov, "Frequency locking to the center of a $532 \mathrm{~nm}$ iodine absorption line by using stimulated Brillouin scattering from a single-mode fiber," Optics Letters, Vol. 31, No. 5, March 1, 2006.

9. Edward V. Browell, Michael E. Dobbs, and,Berrien Moore III, "Airborne Demonstration of a CW Laser System for CO2 Column Measurements," $4^{\text {th }}$ International Workshop on Greenhouse Gas Measurements from Space, CNES HQ, Paris, France, 25-27 June 2007, http://geomon.ipsl.jussieu.fr/meetings_IWGGMS_0706_talks.html

10. James B. Abshire, Haris Riris, Graham Allan, Xiaoli Sun, Michael A. Krainak, Stephan R. Kawa, Jian-Ping Mao, Mark Stephen, John F. Burris, "Laser Sounder for Global Measurements of CO2 Concentrations in the Troposphere from Space," $4^{\text {th }}$ International Working Group on Greenhouse Gas Measurements from Space, CNES HQ, Paris, June 27, 2007, http://geomon.ipsl.jussieu.fr/meetings_IWGGMS 0706 talks.html

11. Pierre H. Flamant, "Atmospheric $\mathrm{CO} 2$ measurements by Lidar: an itinerary from ground to space," $4^{\text {th }}$ International Working Group on Greenhouse Gas Measurements from Space, CNES HQ, Paris, June 27, 2007, http://geomon.ipsl.jussieu.fr/meetings_IWGGMS_0706_talks.html 